\title{
Enlarged Prostate, Chronic Prostatitis and Kidney Stone Disease should be Treated Therapeutically with Dr Allen's Devices, 4th Global Physiotherapy Congress in Italy
}

\author{
Simon Allen., MD, PhD, Academician ${ }^{1 *}$ \\ ${ }^{1}$ Director Fine Treatment, Oxford, United Kingdom \\ *Corresponding Author: Simon Allen, MD, PhD, Academician, Director Fine Treatment, Oxford, \\ United Kingdom. Email: info@finetreatment.com
}

\section{BACKGROUND}

The therapeutic aspects for the treatment of most common urological diseases, such as benign prostatic hyperplasia (BPH) or enlarged prostate, Chronic prostatitis/chronic pelvic pain syndrome (CP/CPPS) and kidney stone disease were discussed at the 4th Euro Global Physiotherapy Congress in December 07-082017 at Rome, Italy. The focus of this event was on science, clinical trials and therapy to improve people's lives. Thermobalancing therapy and Dr Allen's Device, which were invented recently, met all the requirements of high-level discussions among health professionals.

Thermobalancing therapy (TT) provides an exceptional natural treatment method for BPH, CP/CPPS and kidney stones, and Dr Allen's therapeutic devices (DATD) are designed to apply this therapy as to the prostate. TT and DATD received a US patent, confirming that the therapy is unique, and this approach to treatment of human body by own energy has never been used before [1]. Thus, the device can be applied to prostate, see Figure 1, or DATD can be applied to kidneys see Figure 2.

The therapy is based on new understanding of the origin of chronic internal diseases. It has been discovered that all chronic internal diseases have the same root. They start at the capillary level due to pathological capillary activity. Under certain circumstances, namely triggers, capillaries can constrict with development of focus of micro-hypothermia in the affected organs tissue. This leads to another function of capillaries spontaneous expansion in this tissue. It increases pressure in the area. Continuously repeat of this pathological activity of capillaries makes a problem in the affected organ chronic [2].

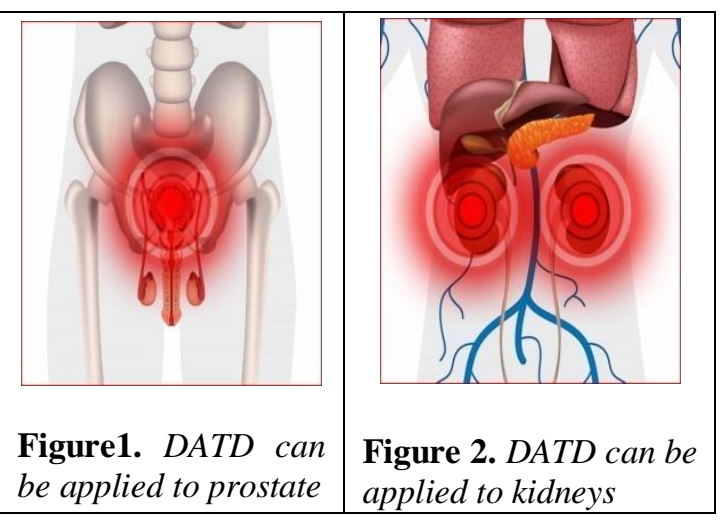

\section{OBJECTIVE}

Observation of TT and DATD studies and empirical evidence in men with chronic nonmalignant prostate diseases and kidney stone disease can confirm the high efficacy of this innovative therapy for prevention and treatment of these chronic conditions[3,4].Furthermore, the investigation shed a light to the etiology and pathophysiology of these health problems.

\section{METHODS AND FINDINGS}

There were two clinical controlled studies undertaken that measured, before and after 6month period, dynamics of urinary symptoms by International Prostate Symptom Score and Quality of Life (I-PSS - QoL) in 124 men with BPH and National Institute of Health Chronic Prostatitis Symptom Index (NIH-CPSI) scores in 45 patients with CP/CPPS (age <55 years), ultrasound - prostate volume (PV) and uroflowmetry- maximum urinary flow rate 
$\left(\mathrm{Q}_{\max }\right)$. Treatment groups received TTand control groups did not. The parameters were compared between groups accordingly. The clinical trials were completed at the Department of Urology of Yerevan State Medical University, the Ethics Committee of which approved the study on TT using DATD. In April 2010, DATD was registered at the Medicines and Healthcare Products Regulatory Agency (UK) as a class-1 medical device. DATD applies a special mixture of waxes (thermoelement) topically to the coccyx area, to the projection of a prostate or kidneys. The thermoelement accumulates the emitted body heat, and thus turn into a source of energy itself. DATD by applying the thermoelement tightly to the skin overcomes the skin barrier and spreads heat energy towards the affected organ.

\section{DISCUSSION}

Evaluation at baseline shows that characteristics of men were identical between treatment and control groups in both studies. The dynamics characteristics has demonstrated that 6-month TT with DATD: (i) reduces pain and improves QoL in patients with CP/CPPS; (ii) reduces urinary symptoms and improves QoL in men with $\mathrm{BPH}$; (iii) reduces $\mathrm{PV}$ and increases $\mathrm{Q}_{\max }$ in both treatment groups with $\mathrm{CP} / \mathrm{CPPS}$ and $\mathrm{BPH}$. We believe that TT is independent treatment for $\mathrm{CP} / \mathrm{CPPS}$ and $\mathrm{BPH}$, as well as for kidney stones, as it dissolves stones gradually. [5, 6,7].

As was seen in both trials DADT gradually reduces the size of enlarged or inflamed prostate gland improving its functioning. It is obviously means that the increased pressure inside the affected organ, i.e. a physical factor, is the actual cause of its disease. These 2 studies suggest that blood circulation plays a key role in the etiology and pathophysiology of BPH and CP/CPPS. The continuous heat exposure that does not exceed the normal body temperature affects the triggers of BPH and CP/CPPS development and progression, i.e. "micro-focus" of hypothermia, and following spontaneous expansion of capillaries $[8,9,10]$.

\section{CONCLUSION}

The theme of the $4^{\text {th }}$ Euro Global Physiotherapy Congress was scientific perspectives for a better living and longer life. TT with DATD reduces pain and urinary symptoms, and dissolves kidney stones gradually. This therapy improves QoL of people without adverse events, promoting healthy longevity. The effectiveness of TT with DATD can be explained by its ability to treat the cause of chronic internal diseases at the vascular level. Thus, TT treats the underlying cause of these chronic diseases. The benefits that people with chronic diseases can get from implementing TT in the global health practice should be considered as soon as possible.

\section{REFERENCES}

[1] Allen, S.; Adjani, A. Therapeutic Device and Method, United States Patent and Trademark Office. U.S. Patent 9,408,744 B2, 9 August 2016. Available online: https://www.google. com/patents/US9408744 (accessed on 20 November 2017).

[2] Allen S The Origin of Chronic Diseases Can Be in Capillary Pathology: An Evidence From Clinical Trials on Thermobalancing Treatment of Prostate Reveals, Achievements in the Life Sciences, 2016, 10, 2, 197-202.

[3] Allen S, Aghajanyan IG. Benign Prostatic Hyperplasia Treatment with New Physiotherapeutic Device, Urol J, 2015 Nov 14; 12(5):2371-2376.

[4] Allen S, Aghajanyan I. Effect of thermobalancing therapy on chronic prostatitis and chronic pelvic pain syndrome, Journal of Clinical Urology, 2016, Sept 20, 1-8, DOI: $10.1177 / 2051415816671036$.

[5] Simon Allen, Thermobalancing Therapy® should be the First-Line Treatment for Kidney Stones and Benign Prostatic Hyperplasia, Discussions at the Medical Conferences. ARC Journal of Urology, 2017; 2(3):1-8 doi: dx.doi.org/10.20431/2456-060X.0203001.

[6] Allen S, Aghajanyan IG New independent thermobalancing treatment with therapeutic device for internal diseases: chronic prostatitis and chronic pelvic pain syndrome, Nephrourol Mon, 2017, e44694. doi: "http://dx.doi.org/ 10.5812 /numonthly.44694" 10.5812 /numonthly .44694 .

[7] Allen S, Aghajanyan IG Thermobalancing conservative treatment for moderate-to-lowdegree lower urinary tract symptoms (LUTS) secondary to prostate enlargement, Cogent Medicine, 2016, 3(1), 1195067.

[8] Allen S, Aghajanyan IG Use of thermobalancing therapy in ageing male with benign prostatic hyperplasia with a focus on 
Enlarged Prostate, Chronic Prostatitis and Kidney Stone Disease should be Treated Therapeutically with Dr Allen's Devices, 4th Global Physiotherapy Congress in Italy

etiology and pathophysiology, Aging Male, 2016,15.http://dx.doi.org/10.1080/13685538.20 16.1247151.

[9] Allen S, Aghajanyan IG Efficacy of Thermobalancing therapy for chronic prostatitis /chronic pelvic pain syndrome, confirmed by clinical study, may suggest ethology and pathophysiology of this disease, Can UrolAssoc J, 2017 Nov 11. doi: 10.5489/cuaj.4473.

[10] Allen, S. The Vascular Factor Plays the Main Role in the Cause of Pain in Men with Chronic Prostatitis and Chronic Pelvic Pain Syndrome: The Results of Clinical Trial on Thermobalancing Therapy.Diseases 2017, 5(4), 25; doi:10.3390/diseases5040025.

Citation: Simon Allen, Enlarged Prostate, Chronic Prostatitis and Kidney Stone Disease should be Treated Therapeutically with Dr Allen's Devices, 4th Global Physiotherapy Congress in Italy. ARC Journal of Urology. 2017; 2(3):15-17 doi: dx.doi.org/10.20431/2456-060X.0203004

Copyright: (c) 2017 Authors. This is an open-access article distributed under the terms of the Creative Commons Attribution License, which permits unrestricted use, distribution, and reproduction in any medium, provided the original author and source are credited. 